\title{
Correction accuracy and collateral laxity in open versus closed wedge high tibial osteotomy. A one-year randomised controlled study
}

\author{
Robert D. A. Gaasbeek • Loes Nicolaas • \\ Willard J. Rijnberg • Corne J. M. van Loon • \\ Albert van Kampen
}

Received: 29 July 2009 /Revised: 8 August 2009 / Accepted: 8 August 2009 / Published online: 27 August 2009

(C) The Author(s) 2009. This article is published with open access at Springerlink.com

\begin{abstract}
In a randomised clinical trial in 50 patients with symptomatic osteoarthritis of the medial compartment of the knee, the clinical results of high tibial osteotomy (HTO) according to the open wedge osteotomy (OWO) and closed wedge osteotomy (CWO) were compared. In both groups locked plate fixation was used. Clinical and radiological assessments were performed preoperatively and after one year. Postoperative hip-knee-ankle (HKA) correction angles were monitored on standing leg X-rays. The effect of HTO on collateral laxity of the knee was measured with a specially designed varus-valgus device. The WOMAC osteoarthritis index, the modified knee society score (KS) and visual analogue scales (VAS) were used to assess symptoms of osteoarthritis, function, pain and patient satisfaction. At one-year follow-up we found accurate corrections in both groups and the planned correction
\end{abstract}

R. D. A. Gaasbeek ( $\bowtie)$

Department of Orthopedics and Traumatology,

Meander Medical Center,

Ringweg Randenbroek 110,

3816 CP Amersfoort, The Netherlands

e-mail: rda.gaasbeek@meandermc.nl

e-mail: gaasbeek@caiway.nl

R. D. A. Gaasbeek · L. Nicolaas · A. van Kampen

Department of Orthopedics,

Radboud University Nijmegen Medical Center,

Geert Grooteplein-Zuid 10,

6525 GA Nijmegen, The Netherlands

R. D. A. Gaasbeek · W. J. Rijnberg · C. J. M. van Loon

Department of Orthopedics, Rijnstate Hospital,

Wagnerlaan 55,

6815 AD Arnhem, The Netherlands angles were achieved. No loss of correction was observed. Furthermore, the medial collateral laxity and the patellar height significantly decreased after OWO. Significant improvements of WOMAC and KS scores were found in both groups. All patients had significantly less pain and were very satisfied with the results. Surgery time was significantly longer in the CWO group, and complications were more frequent in this group. Both techniques led to good and comparable clinical results. The choice of whether to perform an open or a closed wedge osteotomy may be based on preoperative patellar height or concomitant collateral laxity.

\section{Introduction}

Young active patients with medial compartment osteoarthritis of the knee combined with a varus deformity can be treated with a valgus high tibial osteotomy [2, 3, 7, 10, 19]. High tibial osteotomy (HTO) is performed to stop or inhibit progression of osteoarthritis and to avoid or postpone total knee arthroplasty. In order to unload the medial compartment, a valgus producing correction is applied by performing either a closed wedge osteotomy (CWO), or an open wedge osteotomy (OWO). OWO is a relatively new technique and is less involving in terms of surgical technique than $\mathrm{CWO}$, e.g. only one tibial cut needs to be made and osteotomy of the fibula is not necessary [11]. In recent years, with the introduction of new rigid locked implants in combination with new bone-substituting biomaterials, OWO has become more popular than $\mathrm{CWO}$, avoiding co-morbidity associated with the fibular osteotomy. Hypothetically OWO is more 
accurate than CWO. However, in terms of clinical outcome these possible advantages have not yet been thoroughly investigated in well-conducted randomised studies. Furthermore, positioning the osteotomy proximal to the tibial tuberosity and the insertion of the medial and lateral collateral ligament will lead to potential changes in patellar height and collateral laxity. Previous studies which compared CWO and OWO in patients do not answer important clinical questions in terms of outcome, safety and ligamentous stability $[5,11]$.

The aim of this study was to compare OWO and CWO with locked plate fixation regarding the accuracy of the planned correction, the influence on collateral laxity and patellar height and the clinical outcome in a randomised prospective study.

\section{Materials and methods}

\section{Patients}

Fifty consecutive patients suffering from medial compartment osteoarthritis of the knee were included in the study. Between January 2003 and March 2005 they visited the orthopaedic outpatient clinics of the Rijnstate Hospital in Arnhem or the University Medical Centre Saint Radboud in Nijmegen, in the Netherlands. Active patients between 18 and 70 years of age, suffering from medial osteoarthritis of the knee with a hip-knee-ankle (HKA) varus alignment were included. Patients with rheumatoid arthritis or previous osteotomy of the same knee were excluded from this study. All patients gave their informed consent. Approval of the local medical ethics committee was obtained. Patients were randomised for their treatment according to a sealed envelope procedure.

\section{Primary outcome measures}

Achievement of the planned correction angle of an overcorrection of $4^{\circ}$ of the mechanical femur-tibial axis was measured on standing whole leg radiographs. HKA angles were measured by one observer (RG). Medial and lateral collateral laxity of the knee was measured pre- and postoperatively at one-year follow-up and quantified using a varus/valgus testing device [20]. This device consisted of a base plate with a support in which the knee could be fixed in $20^{\circ}$ of flexion in a standardised fashion. The foot was fixed in a mobile shoe with an attached pointer. The varus and valgus laxity was measured by putting a load of $50 \mathrm{~N}$ on the leg which produced a varus or valgus force on the knee which was quantified on a scale on the base plate (Fig. 1). Previously, the intra- and interobserver reliabilities of this device were determined and showed acceptable
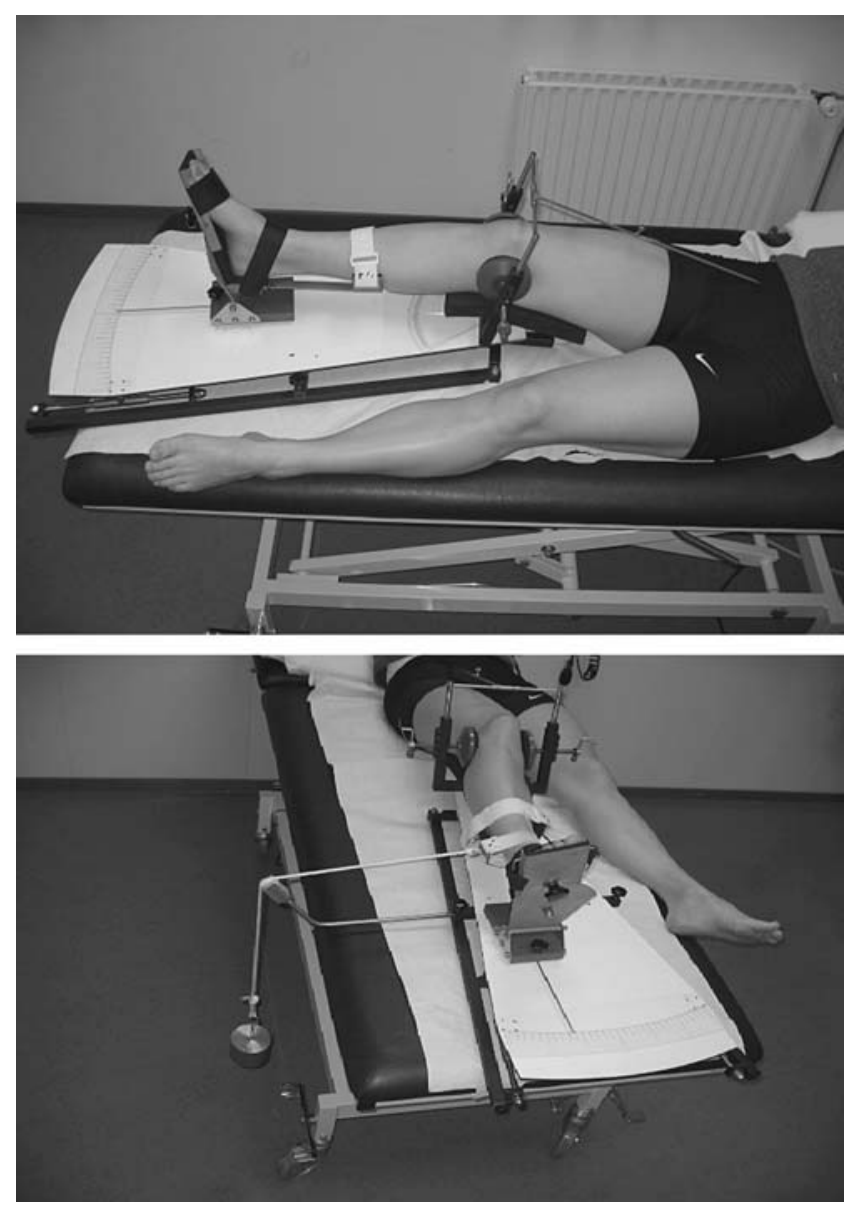

Fig. 1 Measuring the medial and lateral laxity of the knee with the varus-valgus device

intraclass correlation coefficients $(\mathrm{ICC}=0.78,95 \% \mathrm{CI}=$ $0.75-0.79$; ICC $=0.71,95 \% \mathrm{CI}=0.69-0.72$, respectively).

\section{Secondary outcome measures}

Symptoms of osteoarthritis were assessed by the Western Ontario and McMaster Universities (WOMAC) osteoarthritis index. A higher WOMAC score represents worse symptom severity, with 96 points being the worst possible total score. Function was determined using the modified Knee Society score (KS) which assesses pain, range of movement, stability and the ability to walk and climb stairs with 200 points maximum representing the best possible function $[4,12]$. Pain intensity and patient satisfaction were scored with a $0-10$ visual analogue scale (VAS). A high $\mathrm{VAS}_{\text {knee }}$ pain score represents more pain, whereas a low VAS $_{\text {satisfaction }}$ score reveals an unsatisfactory situation for the patient.

Patellar height according to Caton's Index (CI) was measured by one observer $(\mathrm{RG})$ on true lateral radiographic views in $30^{\circ}$ of flexion. Furthermore, surgery time, duration of hospital stay and complications were recorded. 


\section{Statistical analysis}

A multivariable linear regression method was used to analyse the effect of OWO versus CWO on HKA angles, WOMAC, KS and VAS scores, CI, medial and lateral collateral laxity of the knee and complication rate. Age, gender, BMI, and baseline values for the HKA angle were considered as possible confounders and included in the regression model. For statistical analysis the SPSS program (SPSS Inc., Chicago, IL) was used and $p<0.05$ was considered statistically significant.

\section{Operation techniques}

Closed wedge osteotomy (Fig. 2)

A standard anterolateral approach was used. An osteotomy and resection was performed through the fibular head. Two centimetres below and parallel to the joint line a Kirschner wire was inserted to aim the osteotomy. First, the proximal cut was performed and the medial cortex was preserved. Using an aiming device the distal cut was made and the bone wedge was removed. The aiming device we used consists of a blade which is put in the first saw cut. The second cut is performed through a cleft in the device with an adjustable angle to the blade. The defect was closed and the osteotomy was fixed with a four-hole locked plate (Numélock II system, Stryker, Switzerland).

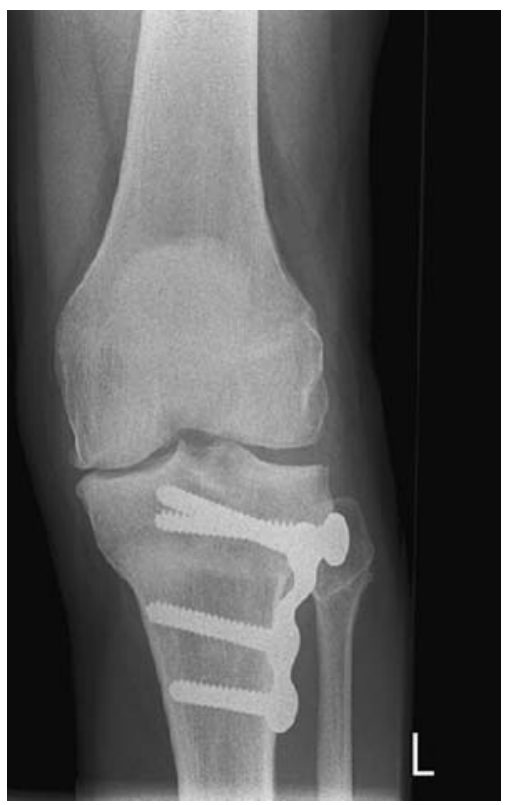

Fig. 2 An example of the closed wedge high tibial osteotomy (CWO)

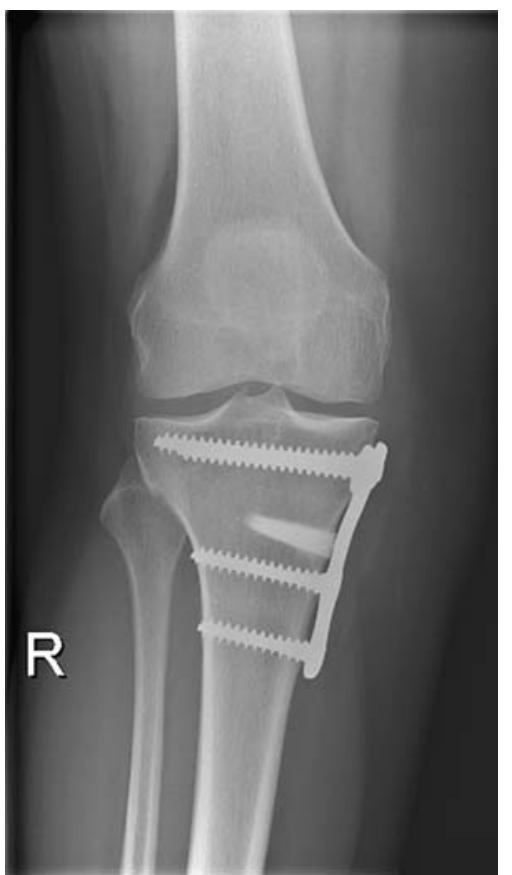

Fig. 3 An example of the open wedge high tibial osteotomy (OWO)

Open wedge osteotomy (Fig. 3)

A medial approach was performed, shifting the pes anserinus and the superficial medial collateral ligament dorsally. A Kirschner wire was inserted parallel to the joint line. Using a guide instrument a second wire was inserted just proximal of the tuberosity in the lateral cranial direction in order to obtain an equal osteotomy angle in all knees. Along this wire the osteotomy was performed. The lateral cortex was left intact. The gap was opened gradually and the preoperatively calculated correction angle was checked with a measuring device. After this the height of the medial gap was measured and the appropriate resorbable tricalcium phosphate (TCP) wedge (Otis, Lourdes, France) was inserted. Internal fixation was performed using a four-hole locked plate (Numélock II system, Stryker, Switzerland).

All surgery was performed under fluoroscopic guidance. All patients received antibiotic prophylaxis preoperatively and postoperative antithrombotic therapy for six weeks. Postoperative management was the same for both groups. Mobilisation started on the first postoperative day with partial weight bearing and full range of motion exercises for six weeks.

\section{Results}

No patients were lost to follow-up. Thirty-six patients were treated in Arnhem (19 CWO and $17 \mathrm{OWO})$ and 14 patients were treated in Nijmegen (6 CWO and 8 OWO). Table 1 
Table 1 Baseline parameters

\begin{tabular}{llll}
\hline Parameter & $\begin{array}{l}\text { Open wedge osteotomy } \\
(n=25)\end{array}$ & $\begin{array}{l}\text { Closed wedge osteotomy } \\
(n=25)\end{array}$ & $\begin{array}{l}\text { Total group } \\
(n=50)\end{array}$ \\
\hline Male/female & $14 / 11$ & $16 / 9$ & $30 / 20$ \\
Age $(y)$ & $47.0(8.5)$ & $49.8(7.4)$ & $48.4(8.0)$ \\
BMI $\left(\mathrm{kg} / \mathrm{m}^{2}\right)$ & $29.7(4.2)$ & $28.4(2.9)$ & $29.0(3.7)$ \\
HKA varus angle $\left({ }^{\circ}\right)$ & $4.3(2.2)$ & $4.1(2.1)$ & $4.1(2.4)$ \\
WOMAC $(0-96)$ & $51.9(18.5)$ & $46.5(14.9)$ & $49.2(16.9)$ \\
KS $(0-200)$ & $111.7(24.1)$ & $113.6(15.9)$ & $112.6(20.2)$ \\
VAS knee pain $(0-10)$ & $6.6(1.7)$ & $6.4(1.3)$ & $6.5(1.5)$ \\
VAS satisfaction $(0-10)$ & $2.3(1.8)$ & $2.8(1.8)$ & $2.6(1.8)$ \\
Medial laxity $\left({ }^{\circ}\right)$ & $5.5(1.8)$ & $5.4(1.2)$ & $5.5(1.5)$ \\
Lateral laxity $\left(^{\circ}\right)$ & $5.2(1.1)$ & $5.2(1.3)$ & $5.2(1.2)$ \\
Caton Index & $1.0(0.2)$ & $1.0(0.2)$ & $1.0(0.2)$ \\
\hline
\end{tabular}

$B M I$ body mass index, $H K A$ hipknee-ankle, WOMAC Western Ontario and McMaster Universities osteoarthritis index, $K S$ Knee Society score, VAS visual analogue scale

Values given as mean (standard deviation)
Primary outcome measures

Table 2 presents the results at one-year follow-up. We found no difference between $\mathrm{OWO}$ and $\mathrm{CWO}$ in the achieved postoperative correction angles. The mean postoperative HKA angle was $3.8^{\circ}$ valgus (SD 2.2) in the OWO group and $4.4^{\circ}$ valgus (SD 2.7) in the CWO group $(p=0.474)$.

The OWO group showed a mean postoperative reduction of the mean medial collateral knee laxity of $4.5^{\circ}$ (SD 1.5) versus $5.3^{\circ}$ (SD 1.2) in the CWO group. This difference was significant $(p=0.041)$. The mean lateral collateral laxity did not change significantly, i.e. $5.3^{\circ}$ (SD 1.1) after OWO and $5.5^{\circ}$ (SD 1.0) after CWO $(p=0.505)$.

Table 2 Results at one-year follow-up

\begin{tabular}{|c|c|c|c|c|c|}
\hline Parameter & $\begin{array}{l}\text { Open wedge osteotomy } \\
(n=25)\end{array}$ & $\begin{array}{l}\text { Closed wedge osteotomy }{ }^{\mathrm{a}} \\
(n=25)\end{array}$ & $\begin{array}{l}\text { Mean } \\
\text { difference }\end{array}$ & $\begin{array}{l}95 \% \text { confidence } \\
\text { interval }\end{array}$ & $p$ value \\
\hline \multicolumn{6}{|l|}{ Primary outcomes } \\
\hline HKA valgus angle $\left(^{\circ}\right)$ & $3.8(2.2)$ & $4.4(2.7)$ & 0.6 & $-0.82 ; 1.93$ & 0.420 \\
\hline Medial laxity $\left(^{\circ}\right)$ & $4.5(1.5)$ & $5.3(1.2)$ & 0.8 & $0.03 ; 1.59$ & $0.041^{*}$ \\
\hline Lateral laxity $\left({ }^{\circ}\right)$ & $5.3(1.1)$ & $5.5(1.0)$ & 0.2 & $-0.39 ; 0.78$ & 0.505 \\
\hline \multicolumn{6}{|l|}{ Secondary outcomes } \\
\hline WOMAC (0-96) & $20.0(19.4)$ & $16.0(15.0)$ & 4.0 & $-13.85 ; 5.85$ & 0.418 \\
\hline $\mathrm{KS}(0-200)$ & $176.6(23.4)$ & $180.2(22.2)$ & 3.6 & $-9.36 ; 16.56$ & 0.579 \\
\hline VAS $(0-10)$ knee pain & $2.5(1.9)$ & $1.8(1.5)$ & 0.6 & $-1 . .60 ; 0.38$ & 0.221 \\
\hline VAS $(0-10)$ satisfaction & $7.8(1.8)$ & $8.7(1.4)$ & 0.9 & $-0.07 ; 1.78$ & 0.07 \\
\hline Caton index & $0.86(0.14)$ & $1.04(0.17)$ & 0.18 & $0.09 ; 0.27$ & $<0.001 *$ \\
\hline Surgery time (min) & $54.8(10.8)$ & $68.0(22.2)$ & 13.2 & $3.27 ; 23.12$ & $0.010^{*}$ \\
\hline Hospital stay (days) & $3.4(1.8))$ & $3.9(1.5)$ & 0.5 & $-0.46 ; 1.42$ & 0.311 \\
\hline
\end{tabular}

HKA hip-knee-ankle, WOMAC Western Ontario and McMaster Universities osteoarthritis index, KS Knee Society score, VAS visual analogue scale

${ }^{\text {a }}$ Values given as mean (standard deviation)

* Indicates a significant $p$ value 
Secondary outcome measures

The results are presented in Table 2. The WOMAC and KS scores showed equal effects between the groups. The mean WOMAC score decreased to 20.0 (SD 19.4) and 16.0 (SD 15.0) after OWO and $\mathrm{CWO}$, respectively. The mean KS score increased to 176.6 (SD 23.4) in the OWO group and 180.2 (SD 22.2) in the CWO group. The mean $\mathrm{VAS}_{\mathrm{knee}}$ pain decreased to 2.5 (SD 1.9) after OWO and 1.8 (SD 1.5) after CWO. In both groups the patients were content with the results after one year. The mean $\mathrm{VAS}_{\text {satisfaction increased to }}$ 7.8 (SD 1.8) in the OWO group and 8.7 (SD 1.4) in the CWO group. There were no significant differences between the groups.

The patellar height significantly decreased after OWO. The small increase of patellar height following CWO was not significant. The mean CI after OWO was 0.86 (SD 0.14 ) and after CWO it was 1.04 (SD 0.17). This difference was significant $(p<0.001)$.

The mean surgery time of $54.8 \mathrm{~min}$ (SD 10.8) in the OWO group was significantly shorter than the mean surgery time for the CWO of $68.0 \mathrm{~min}$ (SD 22.2) $(p=0.010)$. No difference was detected for the mean hospital stay, with 3.4 days (SD 1.8) for the OWO group and 3.9 days (SD 1.5) for CWO.

Complications during follow-up (Table 3)

All patients reported a local hypoaesthesia on the lateral side of the lower leg as a result of cutting branches of the infra-patellar nerve. These findings were the same for both the OWO and CWO groups. One patient in the CWO group suffered a temporary peroneal nerve neuropathy. In the CWO group one case of deep venous thrombosis occurred. One deep wound infection was treated in the CWO group and resolved after surgical lavage and antibiotics.

In this group, two superficial wound infections were successfully treated with antibiotics. In the OWO group, one superficial wound infect was treated with antibiotics. Because of pain the osteosynthesis material was removed in

Table 3 Complications during follow-up

\begin{tabular}{lll}
\hline Complication & $\begin{array}{l}\text { Open wedge } \\
\text { osteotomy } \\
(n=25)\end{array}$ & $\begin{array}{l}\text { Closed wedge } \\
\text { osteotomy } \\
(n=25)\end{array}$ \\
\hline Peroneal nerve neuropathy & - & 1 \\
Deep venous thrombosis & - & 1 \\
Deep wound infection & - & 1 \\
Superficial wound infections & 1 & 2 \\
Removal of osteosynthesis material & 2 & 1 \\
\hline
\end{tabular}

two patients in the OWO group and in one patient in the CWO group.

\section{Discussion}

The purpose of this study was to compare the OWO and the CWO in a randomised clinical setting. Randomised clinical research in this field is very limited. At the time of design of this study, two randomised trials concerning OWO versus CWO had been published by Magyar et al. [16, 17]. However, neither of these studies is directly comparable to this project, since in both studies the OWO was performed by the hemicallotasis technique (HCO) and the CWO was fixed with staples. They found significant loss of correction in the CWO group and a significantly more stable fixation with HCO. Furthermore, they found more complications in the HCO group, mainly pin-track infections. The clinical scores were improved in both groups with no significant difference between the groups. Hospital stay of the HCO group was significantly shorter.

Adili et al. performed a case controlled study of HTO with the Ilizarov external fixator versus the Coventry-type CWO. They found significantly better WOMAC scores but also more complications in the Ilizarov group [1].

Nakamura et al. compared the HCO to the dome osteotomy (DMO) in a randomised clinical trial. The HCO group had significantly less changes in patellar height and tibial slope. The femur-tibia angles were not significantly different [18].

Recently, Brouwer et al. performed a prospective randomised trial in 92 patients. At one-year follow-up they concluded that CWO achieved a more accurate correction and that both OWO and CWO reduce pain and improve function. A relevant difference to our study is the fact that they used an unlocked implant (Puddu, Arthrex, Naples, Florida, USA) in the OWO and staples in the CWO group. Furthermore, a plaster cast was part of the after-treatment [6].

Hoell et al. presented the clinical results of open versus closed wedge HTO techniques in a retrospective study in 108 patients [11]. They analysed the results and outcome of OWO with a Puddu plate versus a Coventry-type CWO with staples. They observed no differences in outcome between the two methods. Both groups showed a significant improvement of clinical scores. A drawback of this study was the non-randomised setting. Furthermore, the statistical analysis was not transparently reported.

Rudan and Simurda [21] found in their study that optimal clinical results were associated with a correction of the femur-tibial angle between $6^{\circ}$ and $14^{\circ}$. Undercorrection was associated with a high failure rate. Overcorrection to a femur-tibial angle greater than $15^{\circ}$ appeared 
to have an even better clinical outcome, but these results were cosmetically unappealing to the patients. Other authors also recommend over-correction of the femur-tibial angle $[13,15]$. Hernigou et al., on the contrary, found that an overcorrection of more than $6^{\circ}$ is associated with progressive degeneration of the lateral compartment and an under-correction is associated with poorer result and reappearance of the medial compartment osteoarthritis [10]. A previous cadaveric RSA study showed that both CWO and OWO gave an acceptable correction with a high variation of postoperative correction angles. This study showed a tendency of overcorrection in the CWO group and no difference in initial stability between CWO and OWO with a rigid locked plate fixation [9].

In this clinical trial the aim was a HKA valgus angle of $4^{\circ}$. We found a postoperative angle of $3.8^{\circ}$ (SD 2.2, range $1.0-10.6$ ) in the OWO group and $4.3^{\circ}$ (SD 2.7, range 1.012.0) in the CWO group. We observed no loss of correction after one-year follow-up. Thus, we conclude that both techniques led to an accurate and reliable correction with locked plate fixation.

In varus osteoarthritis of the knee, often a pseudo medial hyperlaxity is observed due to the loss of medial joint space. Because the osteotomy is located proximally to the superficial medial collateral ligament, opening the osteotomy will (re-) tighten the medial structures. Hoell et al. investigated the influence of HTO on the medial and lateral collateral stability. After OWO they found a shift from second $(5-10 \mathrm{~mm})$ and third degree $(>10 \mathrm{~mm})$ to first degree $(<5 \mathrm{~mm})$ medial instability. In their study, the level of instability was determined by physical examination, which is not very objective [11]. We observed a significant decrease in medial collateral laxity of the knee after OWO. The lateral and medial collateral laxity was measured with a specially developed device which enables objective reliable and reproducible varus and valgus measurements [20]. If the medial collateral ligament is tensioned excessively, e.g. in large corrections, the pressure in the medial joint compartment may increase. To prevent this and to obtain the desired correction the superficial medial collateral ligament should be partially released to allow for proper opening of the osteotomy.

OWO proximal to the tibial tuberosity has been reported to cause patella baja $[5,14,23]$. Closed wedge osteotomy (CWO) has been shown to lead to both patella baja and alta. These alterations in patellar height have been attributed to the proximalisation (CWO) or distalisation (OWO) of the tibial tuberosity following HTO. Furthermore, scarring, adhesions and contracture of the patella ligament are mentioned to cause lowering of the patella in CWO [22]. As expected, we observed that the mean patellar height significantly decreased in the OWO group. We saw no significant change in patellar height after CWO. We advise performing a distal tuberosity osteotomy to prevent distali- sation of the tibial tuberosity with OWO in large corrections or in cases of preexistent patella baja [8].

To our knowledge, this study is the first randomised clinical trial comparing OWO and CWO using rigid locked implants and the same functional postoperative treatment protocol in both groups. At one-year follow-up we found significant improvements of WOMAC and KS scores in both groups. Furthermore, all patients had much less pain and were significantly satisfied with the treatment. There were no differences in outcome between the groups. The medial collateral laxity and the patellar height significantly decreased after OWO.

In literature, a wide range of adverse events have been reported for both the OWO and CWO techniques [24, 25]. Accurate preoperative planning and a meticulous surgical technique with the use of rigid stable implants can minimise the complication rate.

We found more adverse events in the CWO group. Overall, complication rates were low in both groups.

The authors are aware that this study concerns only a short-term follow-up. Longer follow-up is essential to obtain information about the ongoing clinical results in both groups. Nevertheless, this study has shown that both osteotomy techniques led to good and comparable clinical results. Surgery time was significantly longer in the CWO group, and complications were more frequent in this group. In large OWO corrections, the tightening of the medial collateral ligament and lowering of the patella should be addressed by the surgeon. Along with the fact that the OWO procedure is less demanding and that it offers the surgeon more opportunities to vary the amount of valgus and slope correction intraoperatively, the authors prefer the OWO in their clinical practices. In this study OWO showed comparable results to $\mathrm{CWO}$ and it was a safe and reproducible technique. Furthermore, the osteotomy of the fibula along with its rather high comorbidity of pain, pseudoarthrosis and peroneal nerve palsy, is not necessary with OWO. This technique seems to be more predictable for correcting varus deformities of the knee.

Open Access This article is distributed under the terms of the Creative Commons Attribution Noncommercial License which permits any noncommercial use, distribution, and reproduction in any medium, provided the original author(s) and source are credited.

\section{References}

1. Adili A, Bhandari M, Giffin R, Whately C, Kwok DC (2002) Valgus high tibial osteotomy. Comparison between an Ilizarov and a Coventry wedge technique for the treatment of medial compartment osteoarthritis of the knee. Knee Surg Sports Traumatol Arthrosc 10:169-176 
2. Aglietti P, Buzzi R, Vena LM, Baldini A, Mondaini A (2003) A high tibial valgus osteotomy for medial gonarthrosis: a 10- to 21year study. J Knee Surg 16:21-26

3. Amendola A (2003) Unicompartmental osteoarthritis in the active patient: the role of high tibial osteotomy. Arthroscopy 19(Suppl 1):109-116

4. Bellamy N, Buchanan WW, Goldsmith CH, Campbell J, Stitt LW (1988) Validation study of WOMAC: a health status instrument for measuring clinically important patient relevant outcomes to antirheumatic drug therapy in patients with osteoarthritis of the hip or knee. J Rheumatol 15:1833-1840

5. Brouwer RW, Bierma-Zeinstra SM, van Koeveringe AJ, Verhaar JA (2005) Patellar height and the inclination of the tibial plateau after high tibial osteotomy. The open versus the closed-wedge technique. J Bone Joint Surg Br 87:1227-1232

6. Brouwer RW, Bierma-Zeinstra SM, van Raaij TM, Verhaar JA (2006) Osteotomy for medial compartment arthritis of the knee using a closing wedge or an opening wedge controlled by a Puddu plate: a one-year randomised, controlled study. J Bone Joint Surg Br 88:1454-1459

7. Choi HR, Hasegawa Y, Kondo S, Shimizu T, Ida K, Iwata H (2001) High tibial osteotomy for varus gonarthrosis: a 10- to 24year follow-up study. J Orthop Sci 6:493-497

8. Gaasbeek RD, Sonneveld H, van Heerwaarden RJ, Jacobs WC, Wymenga AB (2004) Distal tuberosity osteotomy in open wedge high tibial osteotomy can prevent patella infera: a new technique. Knee 11:457-461

9. Gaasbeek RD, Welsing RT, Verdonschot N, Rijnberg WJ, van Loon CJ, van Kampen RJ (2005) Accuracy and initial stability of open- and closed-wedge high tibial osteotomy: a cadaveric RSA study. Knee Surg Sports Traumatol Arthrosc 13(8):689-694

10. Hernigou P, Medevielle D, Debeyre J, Goutallier D (1987) Proximal tibial osteotomy for osteoarthritis with varus deformity. A ten to thirteen-year follow-up study. J Bone Joint Surg Am 69:332-354

11. Hoell S, Suttmoeller J, Stoll V, Fuchs S, Gosheger G (2005) The high tibial osteotomy, open versus closed wedge, a comparison of methods in 108 patients. Arch Orthop Trauma Surg 125(9):638-643

12. Insall JN, Dorr LD, Scott RD, Scott WN (1989) Rationale of the Knee Society clinical rating system. Clin Orthop Relat Res 248:13-14

13. Ivarsson I, Myrnerts R, Gillquist J (1990) High tibial osteotomy for medial osteoarthritis of the knee. A 5 to 7 and 11 year followup. J Bone Joint Surg Br 72:238-244
14. Kesmezacar H, Erginer R, Ogut T, Seyahi A, Babacan M, Tenekecioglu Y (2005) Evaluation of patellar height and measurement methods after valgus high tibial osteotomy. Knee Surg Sports Traumatol Arthrosc 13(7):539-544

15. Kettelkamp DB, Wenger DR, Chao EY, Thompson C (1976) Results of proximal tibial osteotomy. The effects of tibiofemoral angle, stance-phase flexion-extension, and medial-plateau force. $\mathrm{J}$ Bone Joint Surg Am 58:952-960

16. Magyar G, Ahl TL, Vibe P, Toksvig-Larsen S, Lindstrand A (1999) Open-wedge osteotomy by hemicallotasis or the closedwedge technique for osteoarthritis of the knee. A randomised study of 50 operations. J Bone Joint Surg Br 81:444-448

17. Magyar G, Toksvig LS, Lindstrand A (1999) Changes in osseous correction after proximal tibial osteotomy: Radiostereometry of closed- and open-wedge osteotomy in 33 patients. Acta Orthop Scand 70:473-477

18. Nakamura E, Mizuta H, Kudo S, Takagi K, Sakamoto K (2001) Open-wedge osteotomy of the proximal tibia hemicallotasis. J Bone Joint Surg Br 83:1111-1115

19. Odenbring S, Egund N, Hagstedt B, Larsson J, Lindstrand A, Toksvig-Larsen S (1991) Ten-year results of tibial osteotomy for medial gonarthrosis. The influence of overcorrection. Arch Orthop Trauma Surg 110:103-108

20. Rasenberg EI, Lemmens JA, van Kampen A, Schoots F, Bloo HJ, Wagemakers HP, Blankevoort L (1995) Grading medial collateral ligament injury: comparison of MR imaging and instrumented valgus-varus laxity test-device. A prospective double-blind patient study. Eur J Radiol 21:18-24

21. Rudan JF, Simurda MA (1990) High tibial osteotomy. A prospective clinical and roentgenographic review. Clin Orthop 251-256

22. Scuderi GR, Windsor RE, Insall JN (1989) Observations on patellar height after proximal tibial osteotomy. J Bone Joint Surg Am 71:245-248

23. Wright JM, Heavrin B, Begg M, Sakyrd G, Sterett W (2001) Observations on patellar height following opening wedge proximal tibial osteotomy. Am J Knee Surg 14:163-173

24. Nelissen EM, van Langelaan EJ, Nelissen RGHH (2009) Stability of medial open wedge high tibial osteotomy: a failure analysis. Int Orthop Feb 3 (Epub ahead of print)

25. Tunggal JAW, Higgins GA, Waddell JP (2009) Complications of closing wedge high tibial osteotomy. Int Orthop Jun 24 (Epub ahead of print) 